\title{
Technè
}

La science au service de l'histoire de l'art et de la préservation des biens culturels

$43 \mid 2016$

Une Europe de la recherche en sciences du patrimoine

\section{Les cuirs dorés polychromes et le multi-accès FIXLAB CHARISMA : genèse d'une recherche innovante}

Gilt leather and CHARISMA's multi-access FIXLAB: genesis of an innovative research

Laurianne Robinet, Céline Bonnot-Diconne, Claire Pacheco, Marie Radepont, Jean-Pierre Fournet, Mariabianca Paris et Marcella loele

\section{OpenEdition}

\section{Journals}

Édition électronique

URL : http://journals.openedition.org/techne/856

DOI : $10.4000 /$ techne.856

ISSN : 2534-5168

Éditeur

C2RMF

Édition imprimée

Date de publication : 1 août 2016

Pagination : 114-119

ISBN : 978-2-7118-6338-9

ISSN : 1254-7867

Référence électronique

Laurianne Robinet, Céline Bonnot-Diconne, Claire Pacheco, Marie Radepont, Jean-Pierre Fournet,

Mariabianca Paris et Marcella loele, « Les cuirs dorés polychromes et le multi-accès FIXLAB

CHARISMA : genèse d'une recherche innovante », Technè [En ligne], 43 | 2016, mis en ligne le 19

décembre 2019, consulté le 25 juillet 2020. URL : http://journals.openedition.org/techne/856 ; DOI : https://doi.org/10.4000/techne.856

\section{(c) (1) () $\Theta$}

La revue Technè. La science au service de l'histoire de l'art et de la préservation des biens culturels est mise à disposition selon les termes de la Licence Creative Commons Attribution - Pas d'Utilisation Commerciale - Pas de Modification 4.0 International. 


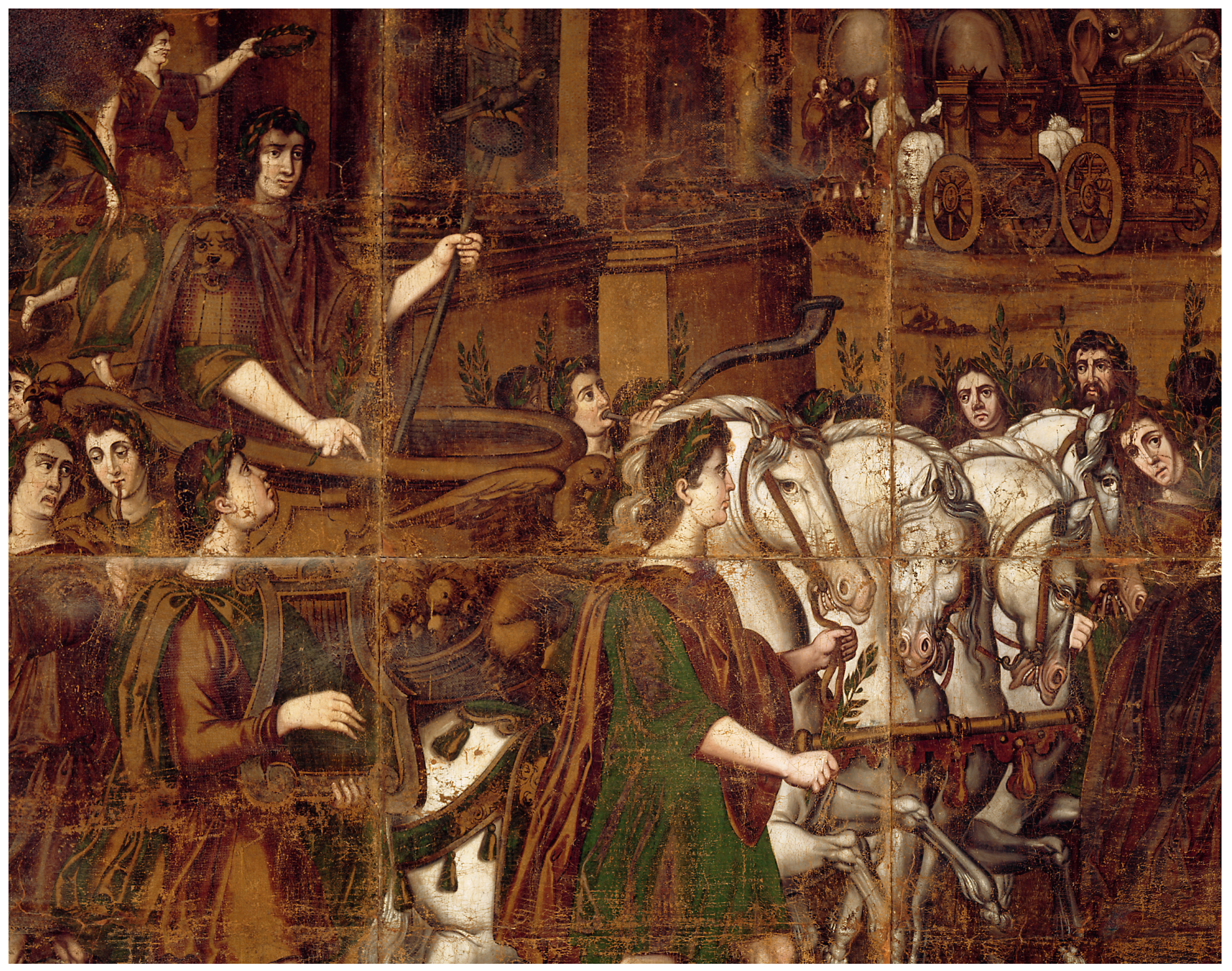

Fig. 1. Le Triomphe de Scipion, d'après Antonio Tempesta, tenture de cuir peint, $\mathrm{XVII}^{\mathrm{e}}$ siècle, musée national de la Renaissance, Écouen. @ RMN-Grand Palais (musée de la Renaissance, château d'Écouen)/Gérard Blot. 
Laurianne Robinet

Céline Bonnot-Diconne

Claire Pacheco

Marie Radepont

Jean-Pierre Fournet

Mariabianca Paris

Marcella Ioele

\section{Les cuirs dorés polychromes et le multi-accès FIXLAB CHARISMA : genèse d'une recherche innovante}

Gilt leather and CHARISMA's multi-access FIXLAB: genesis of an innovative research

Résumé. L'accès à de grands instruments au travers $d u$ programme européen CHARISMA a permis d'initier un programme de recherche sur les cuirs dorés polychromes s'appuyant sur une méthodologie analytique innovante avec une équipe pluridisciplinaire. Ce type de décors, principalement fabriqués entre le XVI et le XVIII ${ }^{e}$ siècle en Europe, est rarement signé. Leur datation et leur provenance sont donc actuellement déduites le plus souvent de leur facture et de leurs caractères stylistiques. L'utilisation combinée des analyses PIXE et RBS sur l'accélérateur AGLAE et de la ligne infrarouge du synchrotron SOLEIL a permis d'analyser les différents composés présents dans ces cuvres : cuir, vernis, feuille d'argent. Les recherches se sont ensuite focalisées sur la caractérisation de l'argent à partir d'un corpus d'échantillons pour tenter de trouver des marqueurs permettant une meilleure classification de ces æuvres et une meilleure compréhension des dégradations observées. L'article expose les principales étapes de cette recherche qui a pour objectif une plus grande connaissance des techniques de fabrication ouvrant vers des identifications plus précises des auvres et de meilleures approches de conservation.

Mots-clés. Cuir doré, argent, provenance, vernis, PIXE, $R B S$, spectroscopie infrarouge synchrotron, cuir de Cordoue.

\begin{abstract}
Access to large-scale facilities through the European project CHARISMA enabled a research program to be launched on gilt leather, involving innovative analytical methodology and a multidisciplinary team. Such decorative leather, produced in Europe mainly between the 16th and 18th centuries, were seldom signed. Nowadays, date and origin are generally deduced from their manufacturing and stylistic characteristics. The combined use of PIXE and RBS analysis on the AGLAE accelerator and the infrared beamline at the SOLEIL synchrotron has enabled researchers to analyse the different components present in these artefacts: leather, varnish, silver leaf. Based on a corpus of gilt leather samples, research then focused on the characteristics of the silver leaf, in an attempt to identify markers that would help classify these decors and shed more light into the deterioration observed. This paper describes the main steps of this research which seeks to broaden understanding of the manufacturing techniques employed and thus help better assign these decors and improve conservation approaches.
\end{abstract}

Keywords. Gilt leather, silver, varnish, manufacturing origin and date, PIXE, RBS, synchrotron infrared spectroscopy, Guadamecil.

\section{Introduction}

Les cuirs dorés, communément mais improprement appelés « cuirs de Cordoue », sont des décors qui ont trouvé leur origine en Espagne il y a un millénaire mais qui ont ensuite été produits dans toute l'Europe, où ils ont connu leur apogée du XVI ${ }^{\mathrm{e}}$ au XVIII ${ }^{\mathrm{e}}$ siècle. Le plus souvent employés comme tentures murales, ils étaient très répandus dans les riches demeures (châteaux et hôtels particuliers notamment) dont ils ornaient les pièces principales (fig. 4). Avec l'apparition des papiers peints au cours de la seconde moitié du XVIII ${ }^{\mathrm{e}}$ siècle, ils passèrent de mode et disparurent alors des intérieurs. Aujourd'hui, presque oubliés, peu représentés dans les collections publiques, ils restent pourtant le témoignage d'un art décoratif luxueux et original, longtemps apprécié dans toute l'Europe. Si, aujourd'hui, les cuirs dorés se sont considérablement raréfiés, ils n'ont cependant pas totalement disparu. Aussi leur étude et leur conservation sont-elles un sujet d'intérêt dans la plupart des pays européens où divers ensembles d'inégale importance sont conservés dans des lieux publics et privés (musées, monuments historiques) ; en France, près d'un millier de décors ont été répertoriés ${ }^{1}$.

L'identification des cuirs dorés (datation et origine) repose essentiellement - puisqu'ils sont rarement signés ou marqués ${ }^{2}$ - sur des éléments parfois incertains, à savoir leur facture et leurs caractères stylistiques. Pour tenter de pallier cette

Laurianne Robinet, ingénieure de recherche, Centre de Recherche sur la Conservation (CRC-CRCC) (lrobinet@mnhn.fr). Céline BonnotDiconne, conservatrice-restauratrice de cuirs, 2CRC, maître d'art, ancienne pensionnaire à la Villa Médicis à Rome (cbonnotdiconne@aol.com). Claire Pacheco, ingénieure de recherche, chef du groupe AGLAE+, département Recherche, C2RMF (claire.pacheco@culture.gouv.fr). Marie Radepont, post-doctorante chimie des matériaux, CRC-CRCC C2RMF (mradepont@mnhn.fr). Jean-Pierre Fournet, historien d'art (j-p.fournet163@orange.fr). Mariabianca Paris, conservatrice-restauratrice de cuirs, Istituto Superiore per la Conservazione ed il Restauro (ISCR), Italie. Marcella Ioele, scientifique, Istituto Superiore per la Conservazione ed il Restauro (ISCR), Italie. 


\section{Schéma de la stratigraphie}

1 Vernis " d'or »

2 Couche de protection

\begin{tabular}{ll}
\hline 4 Colle & 3Feuille d'argent \\
5 Cuir & \\
&
\end{tabular}

\section{b Coupe microtome d'un cuir doré}

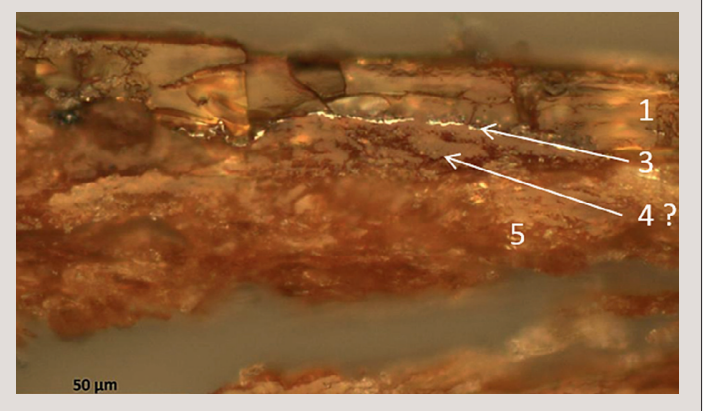

Fig. 2. Schéma de la stratigraphie d'un cuir doré d'après les textes (a), et la stratigraphie visible sur une coupe microtome d'un cuir doré (b). (c) L. Robinet. difficulté, il est apparu nécessaire d'entreprendre des recherches scientifiques ciblées pour accéder à une meilleure connaissance de la composition, des techniques de fabrication et des processus de dégradation de ces œuvres, pour, in fine, permettre une meilleure conservation de ces décors et favoriser leur mise en valeur auprès du public.

Au regard du scientifique, les « cuirs dorés » sont des matériaux complexes à étudier en raison de leur nature composite. Sur l'ensemble du cuir, provenant de peaux de mouton, de chèvre ou de veau, de tannage végétal, sont collées des feuilles d'argent ; celles-ci sont ensuite recouvertes d'un « vernis jaune " fait de résines végétales cuites dans de l'huile de lin, ce qui donne un aspect « doré » (fig. 2) ${ }^{3}$. La surface ainsi obtenue, travaillée au repoussé ou non, est ensuite habituellement décorée avec des peintures à l'huile et ciselée aux petits fers. Les « carreaux » ainsi fabriqués sont assemblés par couture ou collage de façon à obtenir la surface de tenture désirée. Ainsi les cuirs dorés sont-ils à la fois composés de matières organiques (cuir, colle, vernis jaune) et d'une matière inorganique, la couche d'argent.

Les recherches ont été menées en plusieurs étapes au sein de trois programmes de recherche successifs : le projet soutenu par CHARISMA qui a permis de mettre en place une méthodologie pour l'analyse des différents composants des cuirs dorés ; le projet CORDOBA visant à caractériser la feuille d'argent pour tenter de remonter à la provenance des décors ; et enfin le projet CORD'ARGENT qui examinera les processus de dégradation de l'argent.

\section{Mise en place de la méthodologie pour l'analyse des différents composants des cuirs dorés : le projet soutenu par CHARISMA}

La recherche initiale a été impulsée à Rome en 2011-2012 par Céline Bonnot-Diconne - alors pensionnaire à la Villa Médicis -, bientôt rejointe par Mariabianca Paris de l'Istituto Superiore per la Conservazione ed il Restauro (ISCR). Le sujet portait sur l'étude scientifique et technique de la feuille d'argent entrant dans la composition des décors en cuir doré polychrome du XVI ${ }^{\mathrm{e}}$ au XVIII ${ }^{\mathrm{e}}$ siècle en France et en Italie. L'objectif poursuivi était de comparer deux types de production, l'une française et l'autre italienne, pour savoir s'il existait des techniques de fabrication propres à chacun des pays. Dans ce but, un corpus d'une quarantaine de décors en cuir doré français et italiens, provenant d'institutions et de musées européens divers, a été constitué. Il s'agissait de tentures murales ou de devants d'autels n'ayant pas subi de restauration, majoritairement attribués de façon certaine à des fabricants connus grâce aux sources archivistiques.

Pour conduire les analyses approfondies, une demande a été faite à la plateforme FIXLAB de CHARISMA en sollicitant l'accès aux deux grands instruments susceptibles de donner les informations sur les divers constituants de ces œuvres : cuir, colle, feuille d'argent, couche de protection et vernis. La première étape a consisté à définir une méthodologie analytique pour caractériser les différents matériaux, et donc à installer un solide partenariat entre les différents protagonistes et les équipes de recherche d'IPANEMA au synchrotron SOLEIL, et d'AGLAE au C2RMF. La méthodologie devait prendre en compte la complexité du matériau formé de couches successives et la coexistence de composés organiques et inorganiques ; il fallait aussi tenir compte du fait que certains des marqueurs chimiques et/ou technologiques pouvaient n'être présents qu'en faible concentration, en couche très fine, voire à l'état de traces. Par conséquent, il était nécessaire d'utiliser des techniques micro- ou non-destructives, de haute sensibilité et de haute résolution spatiale.

Pour caractériser la feuille d'argent, les cuirs dorés ont été analysés par faisceau d'ions sur l'accélérateur AGLAE, par PIXE (Particle Induced X-ray Emission) pour la composition chimique, et par RBS (Rutherford Backscattering Spectroscopy) pour l'épaisseur. Ces analyses ont été effectuées de façon non invasive sur les zones où la feuille d'argent était apparente et où le vernis était très fin. Plusieurs zones ont ainsi été examinées sur chacun des décors. Les mesures ont montré que la feuille métallique contenait $99 \%$ d'argent et des traces de cuivre et d'or. L'épaisseur de la feuille d'argent variait de 30 à $600 \mathrm{~nm}$, mais elle était le plus souvent voisine de 100 à $200 \mathrm{~nm}$. Quand la feuille d'argent était ternie, les analyses ont montré que la couche de corrosion contenait essentiellement des chlorures et sulfures d'argent.

La caractérisation des matériaux organiques nécessitait un recours à la stratigraphie (fig. 2a), et par conséquent un prélèvement était indispensable. En raison de la nature des matériaux et de leur sensibilité aux solvants (notamment le 
vernis), une technique de préparation à sec, sans inclusion, a été mise au point de façon à obtenir des coupes transversales de 6-8 4 m d'épaisseur par ultramicrotomie sur la plateforme IPANEMA (fig. 2b). Ces coupes ont été analysées sur la ligne de microspectroscopie infrarouge SMIS du synchrotron SOLEIL. La résolution spatiale offerte par le synchrotron rendait possibles les analyses de la couche de vernis, d'une épaisseur moyenne de 10 à $20 \mu \mathrm{m}$, et de la couche de cuir indépendamment l'une de l'autre. Ainsi la technique a-t-elle permis de confirmer les informations données dans les textes anciens, notamment que le vernis contient un mélange d'huile et de résine. Elle a aussi permis d'accéder, dans certains échantillons, à des informations sur le type de tanin et sur l'état de dégradation du cuir. Cependant, la couche de colle et la possible couche de protection de l'argent n'ont pu être caractérisées par les techniques employées ; elles n'étaient généralement pas visibles car probablement diffusées dans les couches voisines.

Des techniques analytiques complémentaires ont aussi été appliquées en laboratoire, notamment, d'une part, la microscopie électronique à balayage couplée à une sonde d'analyse élémentaire pour voir la répartition des produits de corrosion et déceler la présence de matériaux inorganiques au sein des couches de cuir et vernis et, d'autre part, la chromatographie en phase gazeuse couplée à la spectrométrie de masse pour caractériser de façon plus précise les composants chimiques du vernis. Le détail de la méthodologie développée et les résultats obtenus sur trois échantillons représentatifs ont été présentés au congrès triennal de l'ICOM-CC et publiés en $2014^{4}$.

En raison du temps d'accès limité aux grands instruments, l'ensemble des techniques d'analyse utilisées n'a pas pu être appliqué à la totalité des échantillons du corpus. Cependant, les premiers résultats révélaient des variations dans les caractéristiques de la feuille d'argent, susceptibles d'orienter l'attribution des œuvres vers des sites de production, aussi les recherches se sont-elles poursuivies dans ce sens dans le cadre du projet CORDOBA.

\section{La caractérisation de la feuille d'argent, marqueur possible de provenance des œuvres : le projet CORDOBA}

La recherche s'appuie sur l'hypothèse que les différentes caractéristiques de la feuille d'argent utilisée lors de la fabrication des cuirs dorés, c'est-à-dire ses dimensions, son épaisseur, sa composition chimique, pourraient être propres à un atelier, à un fournisseur, à une zone géographique ou, plus simplement, avoir valeur d'indice. Tel était le fondement du projet CORDOBA, financé pour une année, à partir d'octobre 2014, par la Fondation des Sciences du Patrimoine (LabEx PATRIMA). L'objectif était d'identifier des marqueurs physicochimiques susceptibles d'orienter la classification des décors vers des lieux et des époques de production. L'étude, impliquant les moyens techniques du CRCC et de l'équipe AGLAE, a reposé sur l'application des techniques par faisceau d'ions (analyses PIXE et RBS) pour évaluer l'épaisseur, la composition et la dégradation de la feuille d'argent sur un ensemble de cuirs dorés anciens.

Dans un premier temps, des échantillons modèles ont été préparés selon la technique décrite au XVIII ${ }^{\mathrm{e}}$ siècle par Fougeroux de Bondaroy ${ }^{5}$ en utilisant des feuilles d'argent modernes provenant de trois fournisseurs différents. Comme au XVIII ${ }^{\mathrm{e}}$ siècle, les cuirs dorés modèles ont été réalisés sur des peaux de mouton entières de tannage végétal. Après application de colle de peau sur le cuir humidifié (fig. 3a), des feuilles d'argent ont été déposées les unes à côté des autres (fig. 3b), puis la surface argentée a été brunie. Le vernis jaune, préparé en amont, a été appliqué en deux couches successives (fig. 3c). Afin de conserver une zone " argentée ", le vernis, encore liquide, a été retiré sur la moitié de chacune des peaux (fig. 3d). Ces échantillons ont permis l'optimisation de la méthode d'analyse sur AGLAE et la compréhension de l'influence des caractéristiques de la feuille d'argent sur son comportement et sa dégradation.

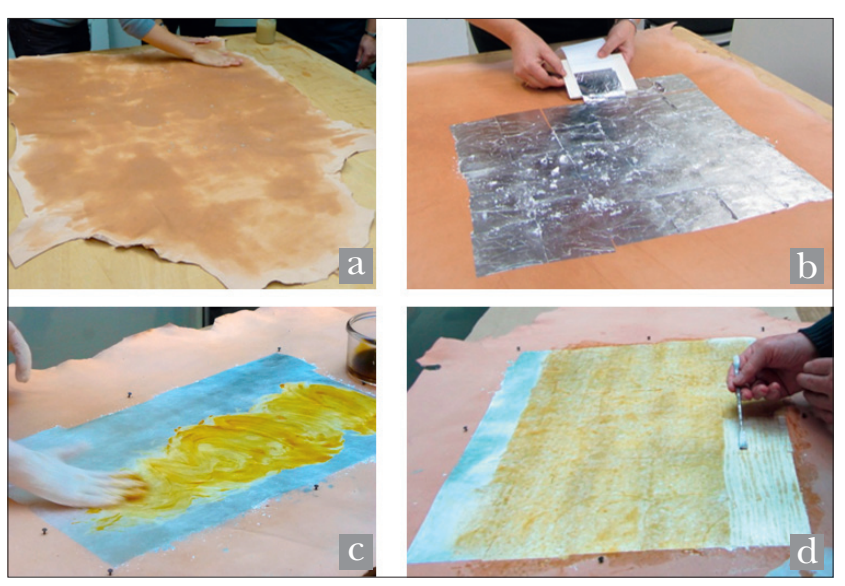

Fig. 3. Étapes successives de la préparation des cuirs dorés modèles, d'après la recette de Fougeroux de Bondaroy (1762). (C) L. Robinet.

En parallèle, l'analyse par faisceau d'ions a été réalisée sur l'ensemble du corpus constitué de 45 échantillons de cuir dorés anciens datant du XVI ${ }^{\mathrm{e}}$ (10 exemples), XVII ${ }^{\mathrm{e}}$ (28) et XVIII ${ }^{\mathrm{e}}$ siècle (5) et originaires d'Italie (23), de France (9), d'Espagne (6) et des Pays-Bas (5). Lors de l'analyse PIXE, de nombreux éléments ont été détectés. Afin de se limiter à la feuille d'argent, il a été décidé de n'étudier que les deux seuls éléments dont la présence est corrélée avec celle de l'argent : l'or et le mercure. La cartographie PIXE, déjà existante sur $\mathrm{AGLAE}^{6}$, permet de voir la répartition spatiale des éléments et d'obtenir des résultats représentatifs de l'objet. C'est d'ailleurs la nécessité d'avoir accès à la répartition spatiale dans l'épaisseur de la feuille d'argent qui a motivé le développement de l'outil permettant une cartographie RBS quantitative. Dans ce cadre, les échantillons modèles de cuir doré ont servi de matériaux de référence pour valider la méthodologie et le traitement automatisé des données (voir l'article de C. Pacheco, D. Gourier et al. dans ce volume). 


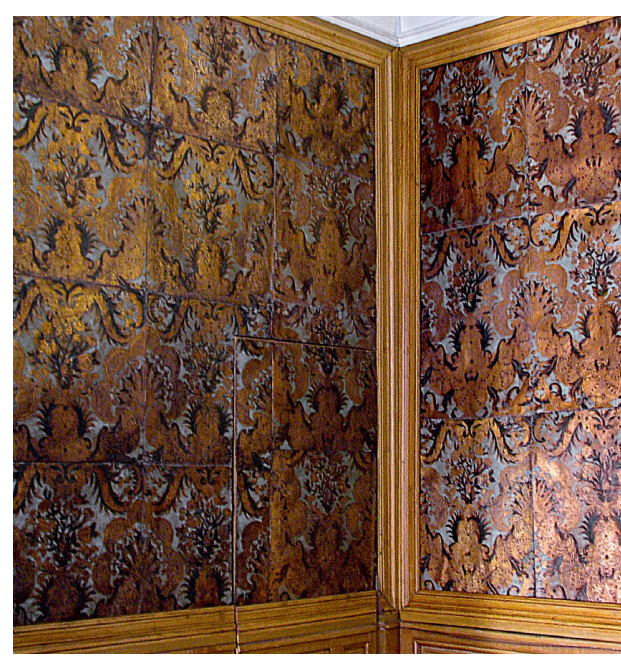

Fig. 4. Tenture murale de l'antichambre, château de Maintenon, Pays-Bas, vers 1740. (C) C. Bonnot-Diconne.

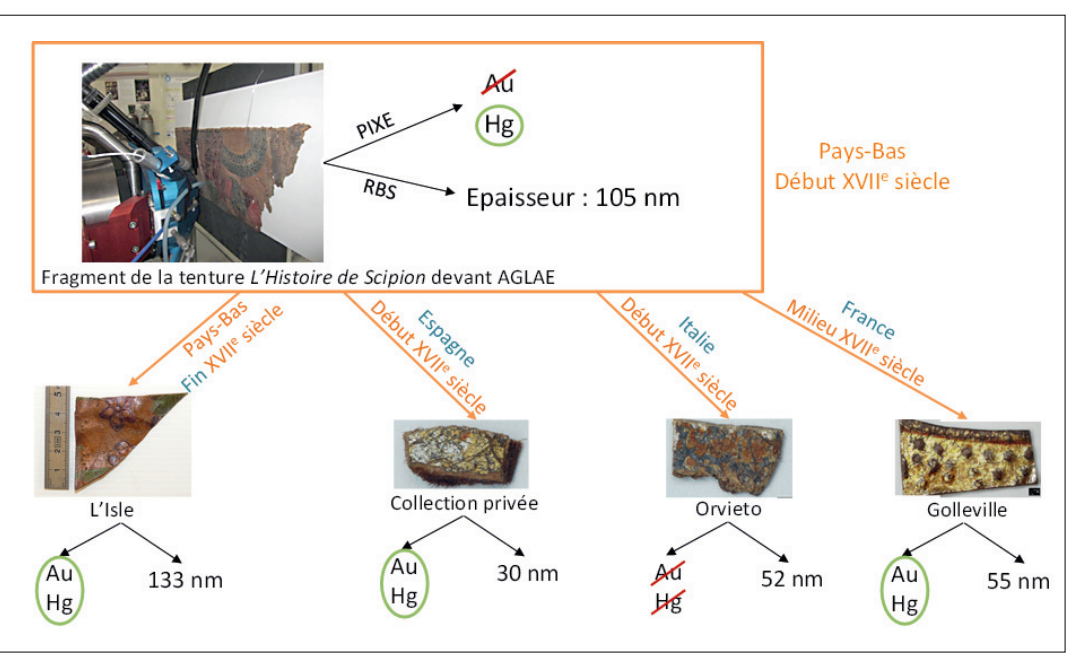

Fig. 5. Comparaison des résultats PIXE et RBS obtenus sur des cuirs dorés du XVII ${ }^{\mathrm{e}}$ siècle d'origines différentes. La présence de mercure $(\mathrm{Hg})$ et l'absence d'or ( $\mathrm{Au})$ constatées dans la tenture flamande du début du XVII ${ }^{\mathrm{e}}$ siècle de L'Histoire de Scipion ainsi que l'épaisseur de sa feuille métallique sont comparées aux mêmes éléments évalués dans des cuirs dorés du XVII ${ }^{\mathrm{e}}$ siècle provenant de différents pays (Pays-Bas, Espagne, Italie, France).
Un fragment non restauré de la tenture de L'Histoire de Scipion du musée national de la Renaissance (fig. 1), fabriquée aux Pays-Bas au début du XVII ${ }^{\mathrm{e}}$ siècle, a été analysé sur AGLAE selon le protocole mis en place. Les premiers résultats des analyses ont montré que la feuille d'argent de cet échantillon contenait du mercure $(\mathrm{Hg})$, mais ne contenait pas d'or $(\mathrm{Au})$ et que l'épaisseur de la feuille était estimée à $105 \mathrm{~nm}$. Ces résultats ont été comparés à ceux obtenus sur d'autres décors originaires du même pays, mais datant de périodes différentes, ou bien sur des décors datant de la même période mais provenant de pays différents (fig. 5). Le traitement des données, notamment RBS, est complexe et long car il repose sur l'utilisation d'outils de simulation, c'est pourquoi sa réalisation est toujours en cours. Cependant, les premières valeurs montrent que la composition et l'épaisseur des feuilles d'argent varient entre les différents échantillons.

À terme, l'étude aura pour objectif de confronter l'ensemble des résultats des analyses physico-chimiques avec les datations et provenances déduites des données stylistiques de chaque pièce du corpus afin de savoir si des marqueurs physiques et/ou chimiques caractéristiques d'une période ou d'un pays peuvent être identifiés. La confrontation devra être plus large et faire intervenir notamment les données, actuellement en cours d'évaluation, concernant la composition des autres éléments constitutifs des œuvres, en particulier le vernis ; l'espèce animale des peaux utilisées (pour lesquelles de nouvelles approches analytiques sont en cours de développement), la nature de la polychromie et des pigments devraient aussi être pris en compte. Il est effectivement probable que ce n'est qu'en croisant l'ensemble de ces informations que l'on peut espérer atteindre le but poursuivi.

En matière de conservation de ces décors, le ternissement de la feuille d'argent est un problème récurrent dont l'origine est encore mal comprise. Lors de la préparation des échantillons modèles, l'étude a mis en lumière que plusieurs paramètres pouvaient avoir une influence sur la corrosion de cette feuille d'argent ; c'est pourquoi des recherches ont été poursuivies dans ce sens dans le cadre du projet CORD'ARGENT.

\section{La corrosion de la feuille d'argent : le projet CORD'ARGENT}

Le ternissement, voire le noircissement, de la feuille d'argent des tentures en cuir doré sont des altérations fréquemment rencontrées dans les collections. Ils sont surtout observés sur les zones qui, à l'origine, ont été laissées argentées et ne possèdent donc en surface qu'une très fine couche de vernis jaune (quelques microns). Ils peuvent se limiter à des zones superficiellement peu étendues mais ils peuvent aussi entraîner un assombrissement généralisé de l'ensemble d'un décor ; dans des situations extrêmes, des plages argentées étendues sont totalement noircies, ce qui affecte profondément la lisibilité de l'œuvre et nuit à l'esthétique de l'ensemble (fig. 6). De façon plus préoccupante aussi, il peut arriver que le ternissement généralisé de la feuille d'argent soit la conséquence d'interventions anciennes de réparation/restauration. Les raisons de cette détérioration ne sont pas toujours clairement établies en raison notamment du manque d'information sur les traitements appliqués et aussi de la complexité et de la multiplicité des opérations de restauration. À l'heure actuelle, il est difficile d'incriminer un produit plutôt qu'un autre (savon, colle, lubrifiant, etc.) ou un procédé plutôt qu'un autre (application de chaleur, humidité, action mécanique, etc. ${ }^{6,7}$. De telles informations seraient pourtant nécessaires pour éviter des erreurs aux conséquences lourdes et irréversibles car l'élimination sans dommage des produits de corrosion de la feuille d'argent est impossible. 


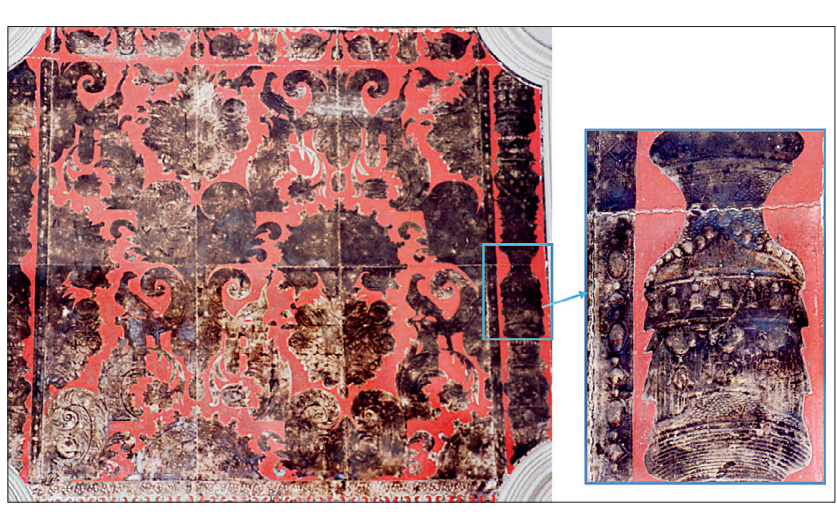

Fig. 6. Décor en cuir argenté polychrome dont les zones « argentées » sont entièrement ternies (collection particulière). (C) J.-P. Fournet.

Sur la base de ces observations, un projet - CORD'ARGENT - centré sur l'étude de la corrosion de la feuille d'argent des cuirs dorés a débuté dans la continuité du projet CORDOBA en collaboration avec l'Institut Lavoisier de Versailles. L'étude devra tenter de déterminer les éléments susceptibles d'être impliqués dans le processus de dégradation et de préciser les mécanismes et la vitesse des réactions mises en jeu ; mais il conviendra au préalable de s'intéresser à deux étapes essentielles de la vie de l'œuvre : sa fabrication et sa restauration. Le projet s'articulera autour de trois axes : l'influence des caractéristiques physico-chimiques propres à la feuille d'argent sur sa réactivité, les interactions entre l'argent et les matériaux organiques constitutifs des cuirs dorés, et l'impact des traitements de restauration. Dans cette étude seront utilisés des échantillons d'argent et de cuir argenté vieillis artificiellement sur lesquels la vitesse d'oxydation du métal sera évaluée ; seront également utilisés des cuirs dorés anciens en cours de restauration sur lesquels l'effet des traitements habituels sera évalué.

\section{Conclusion et perspectives}

La recherche sur les cuirs dorés polychromes, en raison de leur nature composite, mêlant matériaux organiques et inorganiques, a bénéficié de l'accès à deux grands instruments grâce à CHARISMA : AGLAE et le synchrotron SOLEIL. Il est important de noter que ce projet est non seulement le seul ayant obtenu le double accès à ces grands instruments durant la durée du projet CHARISMA, mais également qu'il a généré de solides partenariats permettant d'envisager une suite à ces premiers jalons méthodologiques. Il apparait nécessaire d'élargir encore le champ des investigations, aussi bien pour les périodes considérées que pour les pays concernés de façon à définir un plus grand nombre de marqueurs fiables. Certes, cette étude montre l'importance du développement technologique des grands instruments pour répondre aux problématiques posées par les objets du patrimoine, cependant le déplacement des œuvres ou la prise d'échantillons pour analyse n'est pas toujours réalisable. Il est donc indispensable d'envisager d'autres types d'approches analytiques, par exemple en utilisant des instrumentations portables qui permettraient d'examiner les cuirs dorés directement au sein des collections afin de pouvoir les inclure au référentiel. À terme, le résultat de ces recherches pourrait permettre d'établir une base de données regroupant l'ensemble des cuirs dorés européens dont les caractéristiques - à la fois stylistiques, technologiques et physico-chimiques - seraient aisément accessibles aux spécialistes et responsables des collections.

\section{Remerciements}

Les auteurs souhaitent remercier l'Académie de France à Rome, la Fondation des Sciences du Patrimoine et CHARISMA pour le support financier qui a permis de mener à bien ces recherches. Des remerciements particuliers sont adressés aux personnes ayant contribué à ce travail : Quentin Lemasson, Brice Moignard et Laurent Pichon de l'équipe AGLAE, Éric Laval du C2RMF ; Frédéric Jamme du synchrotron SOLEIL; Loïc Bertrand et Marie-Angélique Languille d'IPANEMA ; Agnès Lattuati-Derieux, Sylvie Thao et Bertrand Lavédrine du CRCC; Roland Février et Marie-Jeanne Dubois de la filière Arts décoratifs du département Restauration du C2RMF ; Marie Heran du 2CRC. Nous remercions Thierry CrépinLeblond, Muriel Barbier et Chantal Bor du musée national de la Renaissance pour l'accueil et l'organisation de la journée d'étude sur les cuirs dorés en octobre 2015, ainsi que les musées, la Conservation des Monuments Historiques et les détenteurs de collections qui nous ont accordé leur confiance.
Notes

1. Fournet, 2004.

2. Cas exceptionnel : la famille Boissier, qui travaille à Avignon entre le XVII ${ }^{\mathrm{e}}$ et le $\mathrm{XVIII}^{\mathrm{e}}$ siècle, élabore un catalogue de leur production que Mr. Fournet a pu relier à des œuvres existantes (voir vol. 3 de Fournet, 2004).

3. Fougeroux de Bondaroy (1762), Schulze (2011).

4. Bonnot-Diconne et al. (2014).

5. Fougeroux de Bondaroy (1762), op. cit. (note 2).

6. Pichon et al. (2014).

7. Bonnot-Diconne et al. (2006).
Bibliographie

Bonnot-Diconne C., Fournet J.P., 2006, La restauration des cuirs dorés. Histoire et techniques, Core, $\mathrm{n}^{\circ} 17$, p. 34-46.

Bonnot-Diconne C., Robinet L., Pacheco C., Ioele M., Paris M., 2014, MultiTechnique Analysis of Gilt Leather Wall-Coverings (16th-18th Century), ICOM-CC 17th Triennial Conference, Melbourne, art. 0701, 8 p.

Fougeroux de Bondaroy A. D., 1762, L'art de travailler les cuirs dorés et argentés, Description des arts et métiers, Paris, Guérin et Delatour, 42 p.

Pichon L., Moignard B., Lemasson Q., Pacheco C., Walter P., 2014,
"Development of a multi-detector and a systematic imaging system on the AGLAE external beam", Nucl. Instrum. Methods B 318, p. 27-31.

Schulze A., 2011, Goldleder zwischen 1500 und 1800 - Herstellung und Erhaltung, Arbeitsheft 17 des Landesamtes für Denkmalpflege Sachsen, Dresden, Ed. Sax-Verlag.

Ouvrage non publié :

Fournet J.-P., 2004, Les cuirs dorés anciens en France, Paris, École du Louvre, Mémoire de Recherche Approfondie, 11 tomes, 1739 p. 